\title{
Les Vannes de toute dernière construction en Suisse
}

\author{
La Vanne-secteur double de l'usine hydroélectrique \\ $R \cup P P E R S W I L-A \cup E N S T E I N$ \\ C. F. KOLLBRUNNER \\ Ingénieur \\ Docteur ès-Sciences techniques - Directeur de la S. A. CONRAD ZSCHOKKE, DOETTINGEN \\ ARGOVIE (Suisse)
}

\section{A. - INTRODUCTION}

Depuis la fin du siècle dernier, la construction des vannes pour barrages s'est développée d'une manière remarquable ( ${ }^{1}$. Vu l'importance des ouvrages hydrauliques en acier, qui représentent

(1) C. F. Kollbruner et J. L. Perrenoud : Dic Entwicklung un Bau von Wehrverschlüsse « Hoch-und Tiefhau $\Rightarrow 1941-N^{\circ} 11,12,13$ ct 17 . une partie essentielle des travaux des construe leurs suisses, les entreprises de construction se sont fortement développées et ont amélioré leurs techniques gràce aux essais minutienx entrepris théoriquement et pratiquement dans ce domaine (Essais en laboratoire).

De la construction des fermetures les plus simples à celle de la vanne perfectionnée de nos jours, on a parcouru, au prix d'un dur effort et

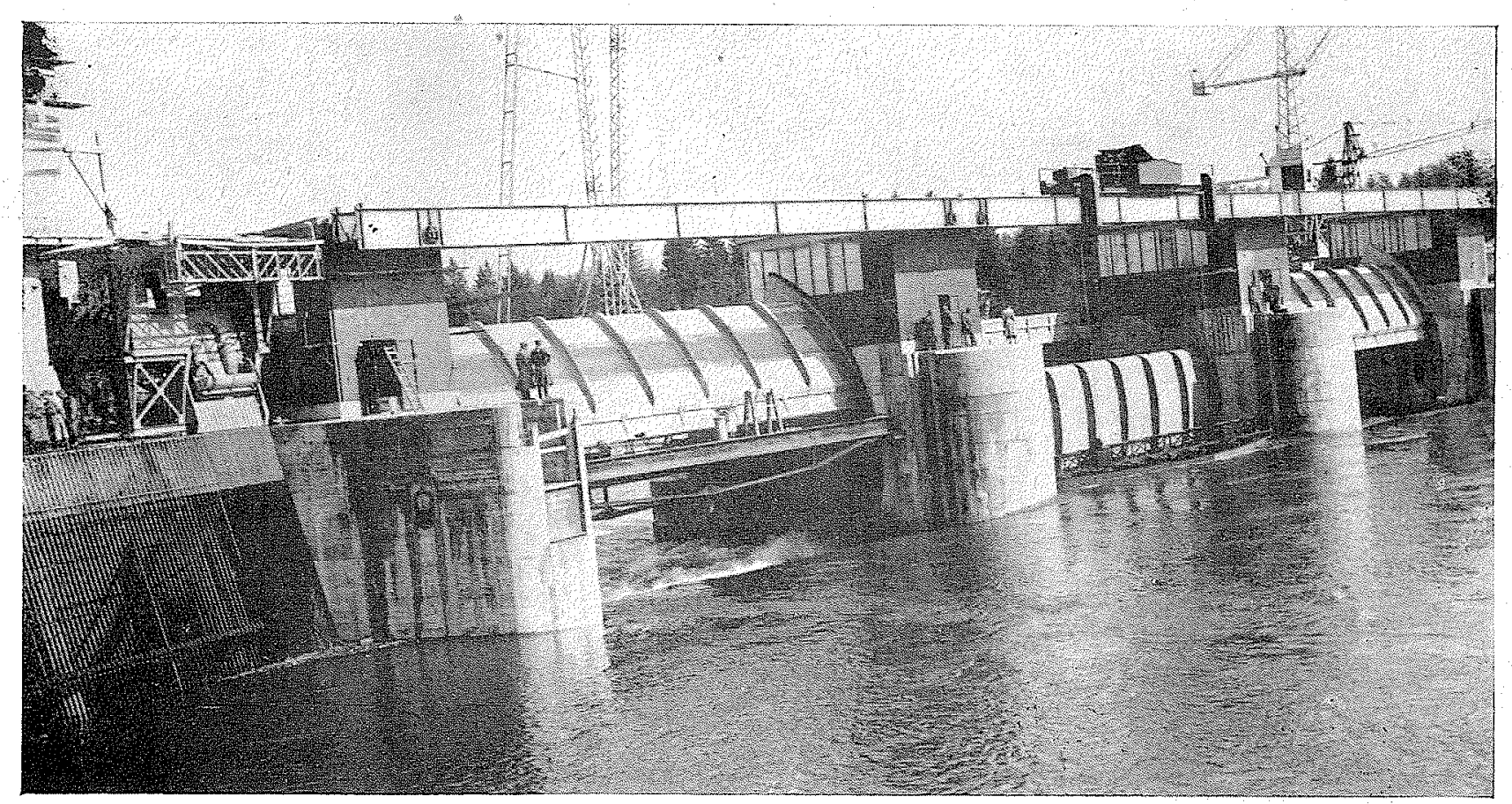

VUE DU BARRAGE DE CÔTÉ AMONT

Passe III (droite) : les deux vannes levées. - Passe II (milieu) : vannes en position fermée. Batardeaux devant les vannes, grues de batardeaux au-dessus des vannes. - Passe I (gauche) : vannes montées sur la passerelle de service. 


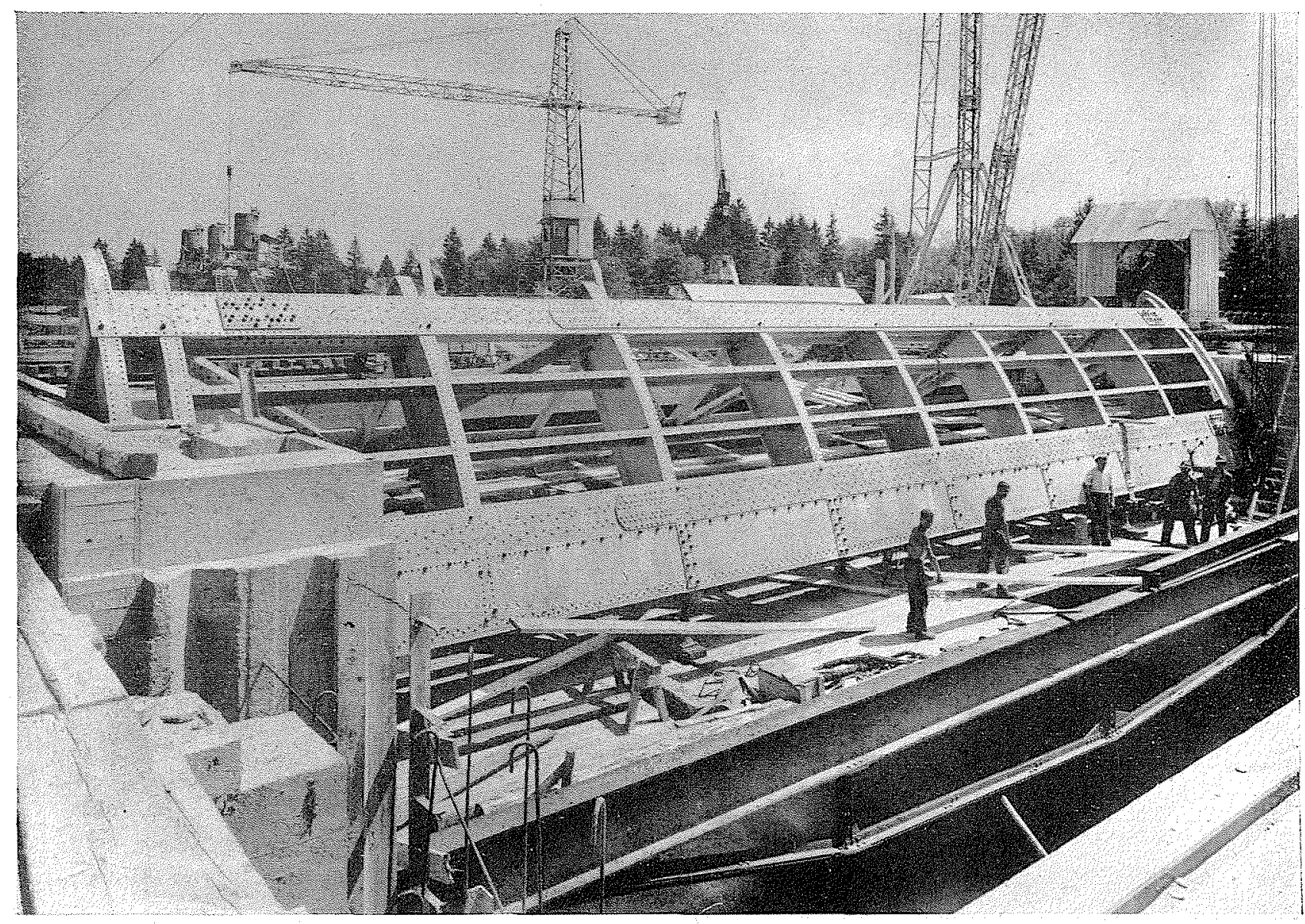

MONTAGE ID JA VANNE INFÉRIEURE, PASSE III

d'une infatigable persévérance, un long chemin, semé de contre-coups et d'obstacles, mais finalement couronné de succès, comme le prouvent les réalisations les plus modernes de la technique. Il va sans dire que ce chemin n'est jamais terminé ; nous visons, en effet, un développement progressif sans cesse adapté aux besoins de l'arenir ; but jamais entièrement atteint, car la construction de chaque usine hydroélectrique exige des études précises et des recherches nouvelles.

Les centrales hydroélectriques importantes ne doivent pas seulement régulariser les cours d'eau et régler les niveaux de retenue en fonction des débits momentanés en utilisant des organes de fermeture d'un fonctionnement rationnel et sûr, et d'un : entretien économique, mais doivent résoudre ce problème en donnant aux ouvrages des formes et dimensions s'harmonisant avec le paysage. Aux considérations d'ordre technique viennent donc s'ajouter des préoccupations d'ordre esthétique. Les vannes ne doivent donc pas être étudiées dans le cadre restreint de leur. réalisation propre, mais en considérant l'ensemble de la construction à réaliser, notamment en ce qui concerne les formes et dimensions à donner à l'infrastructure et à la superstructure des piles qui devront constituer un tout avec la centrale et les ouvrages annexes du barrage.

Alors qu'au commencement du siècle l'on ne construisait généralement que de simples vannes Stonex, on adopta plus tard des vannes doubles de types divers.

Pour l'usine hydroélectrique de RupperswilAuenstein, la S. A. Conrad Zschokke, Constructions Métalliques à Doettingen, se basant sur les progrès réalisés en ce domaine pendant des dizaines d'années et sur de récentes expériences de deux types de vannes à secteur construites par elle pour Verbois-Genève (1940) et LimaPérou (1942), a mis au point une nouvelle vanne, 


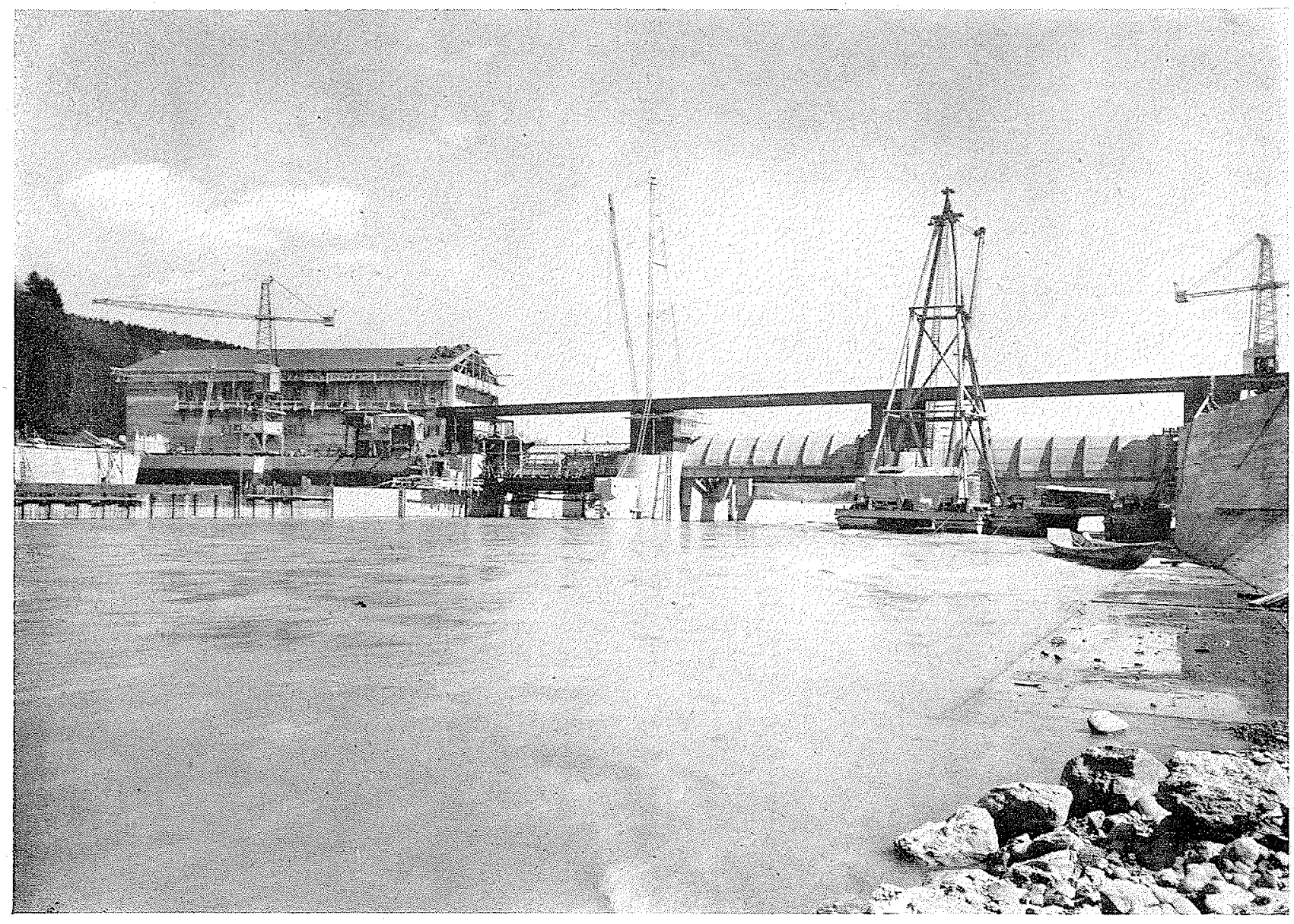

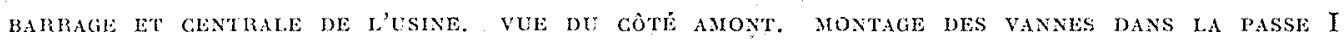

la vanne-secteur double (2) qui représente, vis$\grave{a}$-vis des vannes doubles ordinaires et des vannes à galets avec potence coulissante, des avantages tant techniques qu'économiques. Ce type nouveau de vanne a potence coulissante réunit les avantages de la vanne-secteur, mais ses frais de construction et d'entretien sont bien inférieurs.

Il est bien entendu que la construction de la vanne-secteur double de toute dernière création

(2) C. F. Kollbrunver et J. L. Perrenoud : Die Sektor-Hakenschützen des Kraftwerkes Rupperswil-Auenstein - Schweiz. Bauzeitung vol 123 - No 23 - 1944.

C. F. Konlbrunvin : Die Sektor-Hakenschützen des Kraftwerkes Rupperswil-Auenstein - Schweiz. Wasserkraft und Wasserwirtsehaft, Heft $5 / 61944$.

C. F. Koldinunner : Schuwehrverschlüsse in der tare gestern und heute, Bund. Beilage Technik 10/10/44.

C. F. Kolibnunere : Die Sektor-Hakensehützen. Neue Züreher Zeitung. Beilage Teehnik 29/8/45.

C.. F. Konlununde : Neuartige Wehrversehlüsse : Die Sektor-Hakenschützen des Kraftwerkes Rupperswil Auenstein. Wasser-und Energiewirtschaft Heft $7 / 9 / 45$. C. F. Koldbnenver : La vanne-secteur double pour l'usine hydroélectrique de Rupperswil - Auenstein. Mitteilumgen über Forsehung und Konstruktion im Stahlbau No 3 -1946 - A. G. Gebr. Leemann of C..... Zurich. sortant d'une simple vanne d'autrefois, ne met nullement un point final aux études des vannes de barrages, mais elle demande des recherches ultérieures pour ètre à la hauteur de toute exigence.

En regardant la demière usine à basse chute construite en Suisse, l'usine hydroélectrique de Rupperswil-Auenstein, nous sommes frappés non seulement par la ligne harmonieuse de ses vannes modernes, particulièrement bien adaptées an paysage, mais aussi par leur forme essentiellement rationnelle.

Ce barrage ne possède pas de passerelle des treuils et les vannes sont d'une construction légère ofrrant un aspect esthétique.

\section{B. -- CARACTERISTIOUES TECHNIOUES}

Les caracléristiques techniques sont les suivantes (3) :

(3) Bauleitung des Kraftwcrkes Rupperswil-Auenstein A.G. : Das Kraftwerk Rupperswil-Auenstein. Wasser-und Energiewirtschaft Heft $7 / 8 / 43$. 
Longueur de la retenue utilisée $7 \mathrm{~km} 3$

Débit minimum en basses eaux

(moyenne de 25 ans) .......

Débit utile, moyenne annuelle. .

Débit aménagé $\ldots \ldots \ldots \ldots \ldots$

Débit maximum en hautes eaux

(Janvier 1910) ............

Chute nette aux turbines ..... 9 m a $12 \mathrm{~m}^{3}$

Chute nette moyenne aux turbines $10 \mathrm{~m} 75$

Production moyenne annuelle

d'énergie (deux turbines de

$23.000 \mathrm{cv}$ chacune) .......

$120 \mathrm{~m}^{3} / \mathrm{sec}$.

$275 \mathrm{~m}^{3} / \mathrm{sec}$.

$350 \mathrm{~m}^{3} / \mathrm{sec}$.

$1.300 \mathrm{~m}^{3} / \mathrm{sec}$.

210 millions

de kwh

Cote de la retenue ...... 359 m 60 altitude Cote du seuil du barrage.. $351 \mathrm{~m} 60$ altitade

\section{C. - BARRAGE}

Le barrage de Rupperswil-Auenstein est à trois ouvertures ayant chacune $22 \mathrm{~m}$. de largeur; le niveau d'accumulation est à la cote 359,61$)$ d'altitude et à $8 \mathrm{~m}$. au-dessus du seuil du barrage. Chaque ouverture de barrage est obstruée par une vanne-secteur double. Cette vanne brevetée se compose d'une vanne inférieure normale à secteur et d'une vanne supérieure à seeteur ayant une section en forme de potence.

La vanne inférieure est munie, vers le bas, de renforcement longitudinaux et transyersaux pour assurer sa rigidite; face amont elle possède un revètement en tóle lisse avec barres veriicales. Des crochets de retenue de la vannu supérieure s'accrochent dans ces barres et empèchent ainsi la vanne supérieure de se détacher de la vanne inférieure.

Il $y$ a lieu de souligner que les galets de la vanne supérieure ne peuvent théoriquement jamais être sollicités par des pressions négatives et que le système d'acerochage n'a été prévu que poul les cas accidentels de charges défavorables. Le tablier de la vanne supérieure n'est renforcé verticalement que vers l'amont; sa partie infi.. rieure se recourbe sur toute sa largeur et prend ainsi l'allure d'un coffre dans lequel tournent les galets; la tóle de la partie supérieure à la formt: d'un déversoir. La forme à donner à cette tole pour ètre avantageuse du point de vue hydrau lique, doit ètre choisie de manière à ce que, lors du déversement, la nappe d'eau n'atteigne aucune partie de la vanne, que la charge soit réduite a son minimum et que les dépressions soient éliminces. Etant domné la rotation de la vanne, la forme du déversoir déterminée selon Escinds donne pour chaque position une surcharge d'eau minimum, de sorte que les forces à mettre en cuvre pour monter la vanne sont sensiblemeni réduites. Latéralement, la nappe déversante est dirigée par les tôles de protection. Toute la charpente métallique de la vanne inférieure, telle que poutrelles, couples et entretoises étant disposée sur la face aval, ceux de la vanne supérieure, en revanche, vers l'amont il résulte que la vanne supérieure peut coulisser devant Ia vanne inférieure.

Les couples sont à àmes pleines et partiellement soudés. La vamne inférieure est raidie par deux poutres en treillis rivées, à membrures parallèles, la vanne supérieure par une seule. Ces poutres constituent avec les deux jambages d'appui, un cadre très rigide. Les jambages de la vanne inférieure se rejoignent au pivot des vannes et s'y appuient, ainsi que le jambage double de la vanne supérieure. Ce pivot est réalisé par un robuste palier en fonte d'acier ancré dans une console en béton armé faisant corps avec la pile et placée au-dessus des plus hautes eaux.

Alors que, comme décrit ci-dessus, la vanne inférieure s'appuie sur deux paliers, la vanne supérieure s'appuie, d'une part sur ces paliers, et d'autre part sur la vanne inférieure, par l'intermédiaire de galets fixés à l'extrémité recourbée de la vanne. Gràce à ce dispositif, la pression hydraulique totale correspondant à un pertuis n'est transmise aux piles que par deux paliers mobiles et facilement accessibles.

Le renforcement longitudinal supérieur de la vanne inférieure porte la garniture d'étanchéité intercalaire horizontale; le renforcement infé. rieur, un robuste fer plat, constitue le dispositif d'étanchéité avec le seuil. Les garnitures d'étanchéité verticales sont en «tòle-élastique ». Toutes les pièces et parties mobiles, comme les dispositifs d'étanchéité et les pivots sont munis d'éléments de chauffage électrique leur permettant de jouer en toute saison.

Toute la conslruction des vannes se composant de profils simples et très accessibles, lentrotien en est facile et peu coùteux. Les chaînes servant à soulever les vammes sont fixées a des suspensions basses; conduiles par des guidages spéciaux, elles s'adaptent à la forme des vannes: suspensions et chaînes sont logées dans des niches protégées, aménagées derrière les dispositifs d'étanchéité verticaux; bien qu'étant audessous du niveau de l'eau, elles n'entrent pas en contact avec l'eau dans la position de vanne fermée.

Ce n'est qu'exceptionnellement que la poutrelle principale inférieure de la vanne inférieure arrive à se touver immergée ou arrosée, mais. même alors en cas de déversement par-dessus la vamne, elle est en dehors de la zone critique. On réalise ainsi un élément de barrage absolument irréprochable au point de vue hydrau- 
lique, dont sont éliminés tous les inconvénients constatés souvent dans les autres vannes, tels que : vibrations, flottements et balancements.

Les poids par pertuis sont les suivants :

- vanne supérieure ........ 51 T.

- vanne inférieure ........ $73 \mathrm{~T}$.

- acier coulé ........... $5 \mathrm{~T}$.

Toutes les parties en acier des vannes ont élé protégées contre la rouille par un zingage de $1.000 \mathrm{gr} / \mathrm{m}^{2}$ appliqué au pistolet, c'est-à-dire

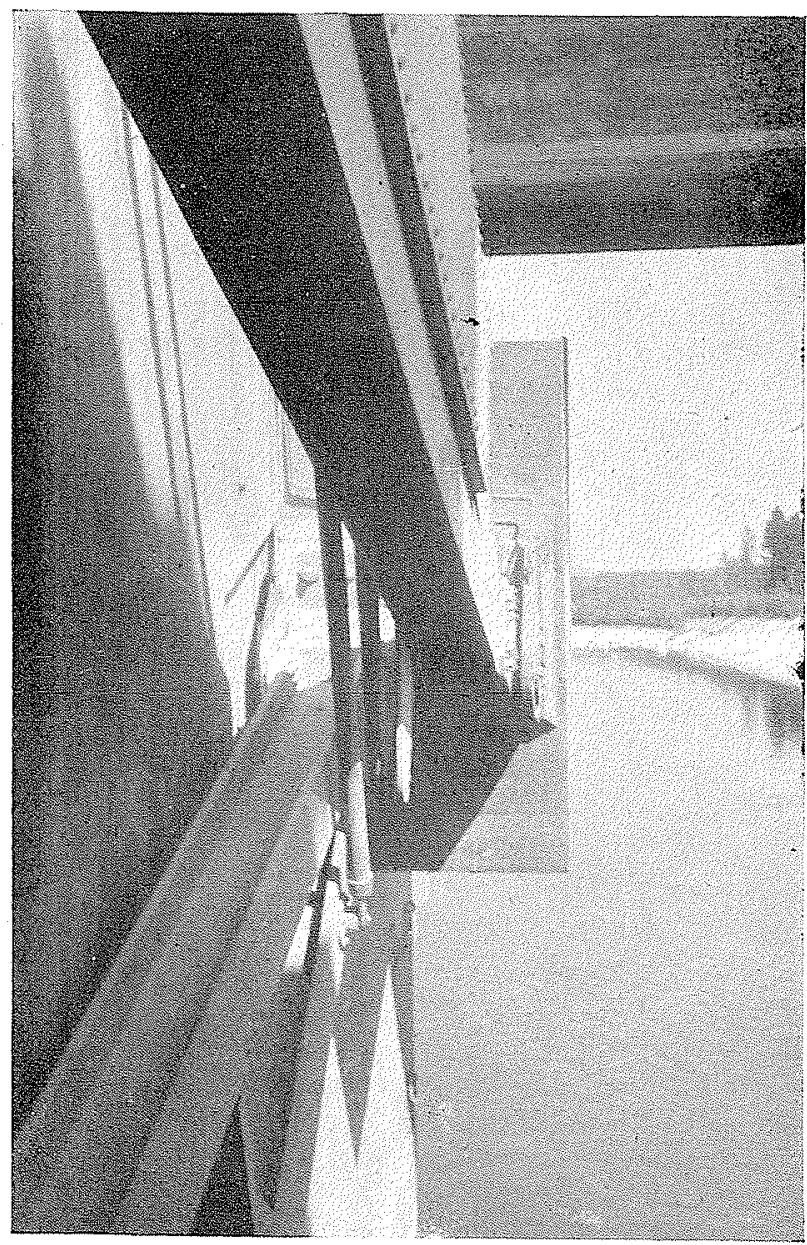

CONSTBUCTION EN BETON ARMÉ AVEC APPUI ET JAMBGES b'APUI, Y COMPRIS IE MÉCANISME DE L'INDICATEVR A DISTANCI

une quantité de zinc de $1.600 \mathrm{gr}$. par $\mathrm{m}^{2}$ de surface a elé jetée par le pistolet dans les ateliers S. A. Conrad Zschokke, à Doettingen, de sorts que, suivant le controle à l'aide d'un instrument d'inspection, une couverture de zine de 1.000 $\mathrm{g} \mathrm{l} / \mathrm{m}^{2}$ au minimum restait sur toute la construction métallique. Les résultats de ce procédé prouvent que le zingage au pistolet représente une couverture plus avantageuse et supérieure a toute peinture.

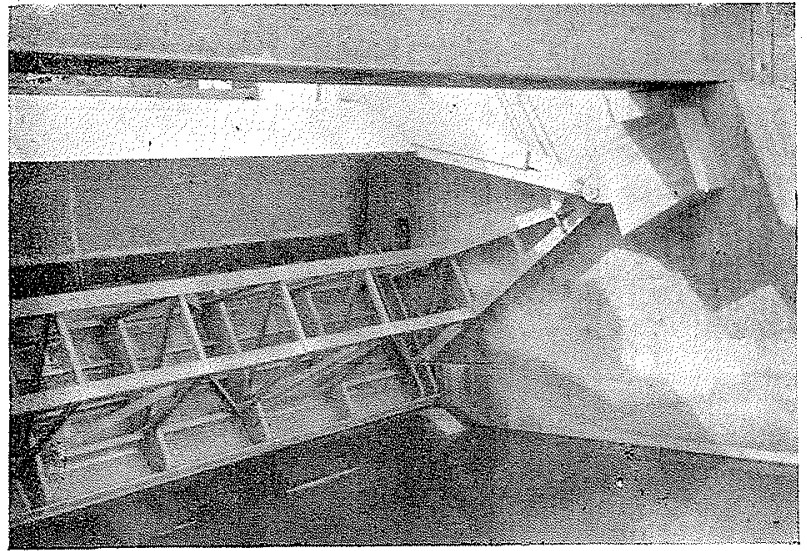

VANYES remMítes. YUE DE Côté AVAL

L'aménagement, la disposition et les dimensions des piles et des superstructures ont été fortement influencés par le choix des nouvelles vannes. A noter que les piles, contrairement aux solutions en usage jusqu'ici, n'ont plus de rainures pour les galets de roulement et ont été concues larges à l'amont et effilées à l'aval, cette dernière partie comprenant deux fortes consoles pour soutenir les pivots. Les piles réalisent, au point de vue hydraulique d'excellentes conditions d'écoulement des eaux et une répartition des masses avantageuses pour leur stabilité. Pour le calcul de l'armature du pilier, ce dernier est. considéré comme étant une plaque; il y a lien de tenir compte des excentricités qui résultent des réactions unilatérales des vannes (t).

Nous avons déjà signalé que les piliers n'on' plus que des rainures pour les batardeaux, les rainures pour les vannes étant supprimées;

(4) C. F. Tioldiruner et Ch. Duzas : Anwendung von Differenzengleichungen zur, Berech von EisenbetonWehepfeilern. Schweiz. Bauzeitung Vol. 124 - $\mathrm{N}^{\circ} 15$ $7 / 10 / 45$.

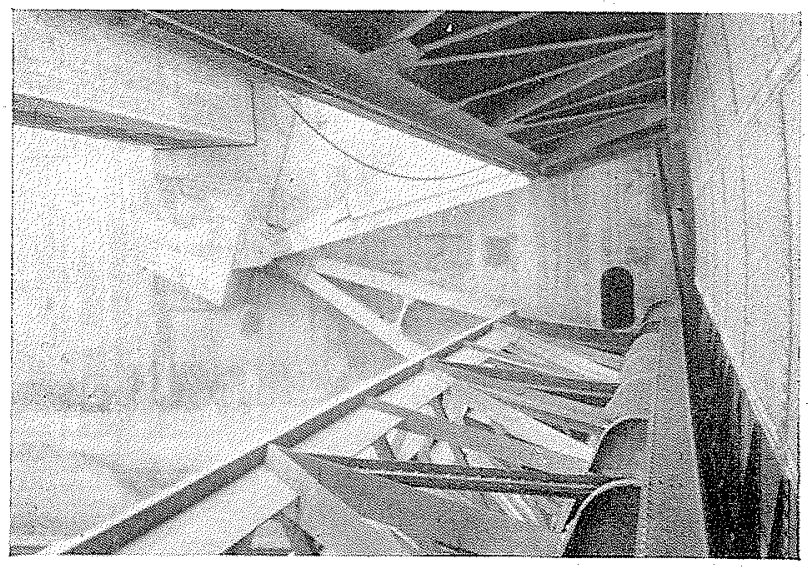

VANES TERMËES, VUE CONTRE LE PIEIER 
ainsi se trouvent éliminés tous les gros inconvénients inhérents aux niches pour les différents trains de galets à rouleaux des vannes Stoney, tant en ce qui concerne les tourbillons le long des piliers que pou'r la construction, l'exploitation, l'entretien et les frais de construction. La suppression des coûteux paliers, chariots, rouleaux et chemins de roulement permet de réaliser de notables économies. En outre, l'emploi d'un seul pivot diminue sensiblement les frottements et l'usure des pièces. Les pivots en acier. coulé de la vanne supérieure et de la vanne inférieure tournent sur des coussinets de bronze et sont lubrifiés à l'aide de presses à haute pression.

Chaque vanne étant actionnée par son propre treuil indépendant, il est facile de réaliser toutes les combinaisons d'écoulement de l'eau par-dessus ou par-dessous les éléments mobiles du barrage. La détermination des forces nécessaires pour lever les vannes a démontré que la vanne-secteur double est aussi plus économique que la vanne à galets en ce quiu concerne l'exploitalion. La résultante des charges se trouvant entre le point de suspension des chaines et le pivot, les charges verticales sont partiellement absorbées par les paliers et coussinets. En outre, étant donné que le bras de levier est très long, le frottement est beaucoup moins grand que dans les vannes à galets. Les appareils de levage des treuils ont donc pu être conçus par une force de levage plus faible que cela aurait été nécessaire pour les autres types de vannes de mème dimension.

Les mécanismes ayant pu être aménagés en profondeur, dans le corps des piles et dans des petites cabines, le constructeur a pu supprimer l'habituel pont ou passerelle des treuils, superstructure toujours inesthétique, qui alourdit le barrage et rompt la pe'rspective de la vallée. Seule une légère poutrelle, portant le chariot de manutention des batardeaux, souligne la construction. On accède aux piliers et cabines des t'reuils par une passerelle en béton armé au niveau du couronnement du barrage; cette passerelle est utilisée comme galerie des câbles et abrite les arbres de transmission des appareils de levage. Ia suppression de la lourde passerelle des treuils permet, d'une part, de réaliser une très importante économie dans les frais de construction et elle donne, d'autre part, au barrage, une silhouette légère qui s'intègre sans heurt dans le paysage.

\section{D. - MONTAGE}

Les opérations de monlage ont été effectuées sur des échafaudages soutenus par des profilés I ancrés dans les piliers. Pour amener à pied d'ocuvre les pièces de construction, on utilisa les ponts de service ayant servi pour les travaux de Génie Civil de l'infrastructure et deux derricks. Les raccords de montage ont été retraités au jeu de sable sur place, puis zingués au pistolet.

\section{E. - DISPOSITIF D'ETANCHEITE}

A la construction de chaque vanne de barrage les dispositifs d'étanchèité jouent un ròle rema'rquable. Ils servent à fermer aussi bien que possible les joints entre les corps mobiles du barrage et les piliers fixes, ainsi qu'entre les différentes parties du barrage elles-mèmes. Vu l'impossibilité d'obtenir une étanchéité complète tout de suite après la fermeture des vannes, il s'agil, pour nous, d'assurer une efficacité maximum grâce à une construction bien concue et ayant fait ses preuves, à l'aide aussi d'une fabrication très soignée et de l'emploi des meilleurs matériaux. De cette facon, il sera possible d'obtenir une étanchéité pour ainsi dire complète.
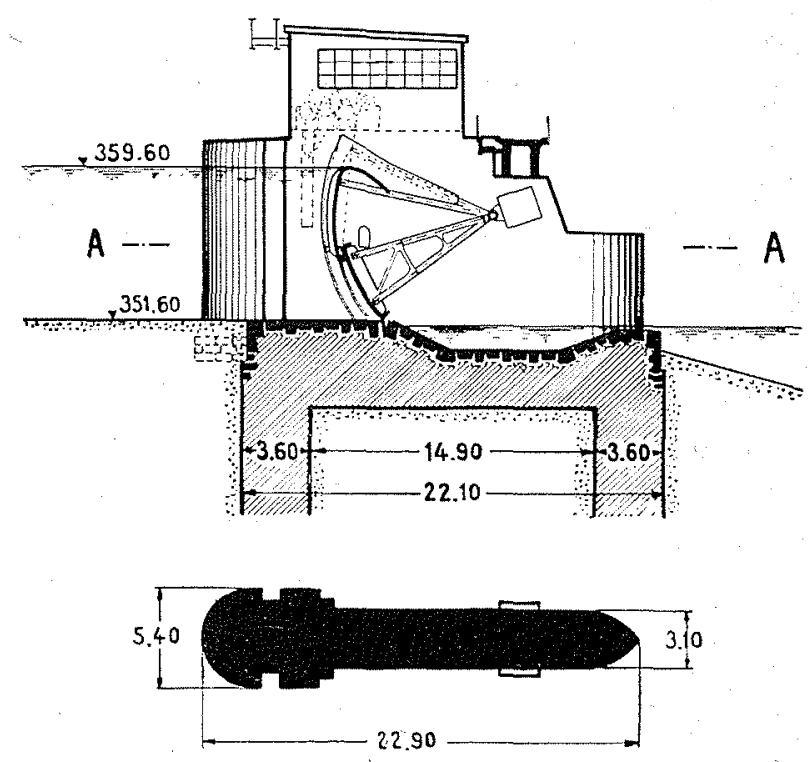

SECTION DU BARRAGE

Etant donné que les vannes sont sujettes à des déformations provenant des diverses charges ou dues à d'autres forces extérieures et qu'elles se dilatent sous l'influence de la chaleur, etc. elles ne se meuvent pas seulement en sens vertical, les dispositifs d'étanehéité doivent être construits de manière qu'ils puissent suivre tous ces mouvements et les contrebalancer, afin que l'eau ne s'infiltre pas par les interstices (5).

(5) C. F. Kordurunner - Stahlwasserbau (Schützen) Stahlbau-Bericht $\mathrm{N}^{\circ} 10$, oct. 46. Edit. par Verband Schweizerischer Brückenbau-und Stahlhochbau-Unternehmungen - Zurich. 
C'étaient surtout les dispositifs d'étanchéité avec le seuil ainsi que la garniture d'étanchéité intercalaire horizontale qui causaient des inconvénients primitifs à l'usine de Rupperswil-Auenstein. Etant donné que ces vannes ne se déforment pas, comme c'est le cas pour les vannes doubles de construction normale, dans le sens de la longueur et de la largeur seulement, mais sur toutes les dimensions, il a fallu cherche'r d'autres constructions pour les étanchements, qui puissent subir toutes les charges et suivre les monvements des vannes. Par conséquent, le dispositif d'étanchéité avec le seuil a été constitué par un robuste fer plat inférieur formé spécialement et la garniture d'étanchéité intercalaire horizontale a reçu un dispositif supplémentaire en tôle élastique et en cuir.

Les essais faits au mois de Décembre dernier dans une des passes à Rupperswil-Auenstein ont prouvé que les dispositifs d'étanchéité, même après mouvement des deux vannes, ne laisseni passer qu'une quantité d'eau inférieure à $2 \mathrm{l} / \mathrm{sec}$. et par $m$., donc une quantité inférieure à cellc fixée au contrat. Quel que soit le débit d'eau, les étanchéités se rétablissent en peu de temps, de sorte que tous les joints sont entièrement étanches.

\section{F. CONCLUSION}

Des études et des expériences poursuivies pendant des dizaines d'années ont permis de réaliser une vanne satisfaisant aux problèmes que posent : la súreté, la sécurité, le fonctionnement, le rendement économique et l'élégance de forme.

Les principaux avantages de la vanne-secteur double sont: isuppression de la passerelle des treuils, forme rationnelle des vannes, appuis simples, mécanisme de levage moins puissant, piles sans rainures poutr les vannes, construction plus rapide.

M. Georges Darrmus a été élu le 2 décembre 1946, membre de l'Académie dés Sciences au fauteuil de M. Léon Gulllet, décédé.

Nous avons déjà eu l'occasion de rappeler à nos lecteurs la qualité et la variété de l'ouve de M. Georges Darricus à l'occasion de l'attribution, à cet éminent savant et ingénieur, du Prix Henri de PARvirfe, pour ses travaux de mécanique et d'électricité appliquée (r).

Notre Revue est heureuse, aujourd'hui, de s'associer à l'hommage décisif qui vient d'ètre rendu à l'œuvre scientifique et technique de 'M. Georges Darrieus, et Iui présente ses félicitations les plus sincères.

(1) La Hotille Blanche - $\mathrm{N}^{\circ} 1-1946$. 